\title{
Extreme Horizontal Branch Stars in Passively Evolving Early Type Galaxies
}

\author{
Fabiola Hernández-Pérez ${ }^{1}$ and Gustavo Bruzual ${ }^{2}$ \\ ${ }^{1}$ Centro de Investigaciones de Astronomía CIDA, A.P. 264, Mérida, Venezuela \\ email: fhernandez@cida.ve \\ ${ }^{2}$ Centro de Radioastronomía y Astrofísica, UNAM, Campus Morelia, México \\ email: g. bruzual@crya. unam.mx
}

\begin{abstract}
We study the effects of including binary star evolution in population synthesis models. We use the Hurley et al. (2002) code to compute binary star evolutionary tracks, and follow the procedure by Han et al. (2002), in particular, the two 2HeWD merger channel, to form EHB stars from a binary pair. We apply the resulting models to study UV excess ETGs.
\end{abstract}

Keywords. UV upturn: galaxies - Elliptical: galaxies - EHB: stars - Binaries: stars.

Using binary star evolutionary tracks computed with a modified version of the Hurley et al. (2002) code, we add the effects of binaries to standard population synthesis models (e.g. Bruzual \& Charlot 2003). Following the isochrone synthesis scheme, we compute a series of isochrones at different ages which include blue stragglers and EHB stars. The resulting isochrones reproduce the principal characteristics of observed CMD of globular clusters. However, EHB in our models tend to be hotter and less luminous than observed in real CMDs. We derive the integrated spectral energy distributions and the colors corresponding to these populations.

The initial mass function (IMF) controls the number of progenitors, affecting the total number of EHB produced in a model that includes binary stars. Thus, Salpeter IMF models result in half the number of EHB as compared to Chabrier IMF models. Metallicity seems to have little effect on the EHB formation rate in these models. In low Z models, the FUV-NUV color is redder and the NUV-r color is bluer, because blue HB stars contribute to the NUV flux.

We compare our model with a crossmatching sample of $\sim 3400$ early type galaxies from SDSS/DR8-GALEX/GR6. We study the color evolution of model populations and evaluate possible scenarios to explain the UV upturn. Population synthesis models including binary evolution cannot reproduce the observed SED's of all early-type galaxies (ETGs). Models remain in the blue zone of the diagram, and the red sequence cannot be reproduced by a model in which binaries are always present. We conclude that it could happen that binary stars are not the only progenitors of EHB stars, and that standard stellar population synthesis models, with no binaries, still remain a valid tool to study ETGs (Yi 2008). However, more realistic treatments of HB and binary star evolution are needed for a better understanding of the UVX phenomenon in ETGs.

\section{References}

Bruzual G. \& Charlot S. 2003, MNRAS, 344, 1000

Han Z., Podsiadlowski P., Maxted P., Marsh T. \& Ivanova N. 2002, MNRAS, 336, 449

Hurley J., Tout C. \& Pols O. 2002, MNRAS, 329, 897

Yi S. K. 2008, ASP Conference Series, 392, 3 